\title{
Transforaminal epidural steroid injections influence Mechanical Diagnosis and Therapy (MDT) pain response classification in candidates for lumbar herniated disc surgery
}

\author{
Hans van Helvoirt ${ }^{\mathrm{a}, *}$, Adri T. Apeldoorn ${ }^{\mathrm{b}}$, Dirk L. Knol ${ }^{\mathrm{c}}$, Mark P. Arts ${ }^{\mathrm{e}}$, Steven J. Kamper ${ }^{\mathrm{c}, \mathrm{f}}$, \\ Maurits W. van Tulder ${ }^{\mathrm{c}, \mathrm{d}}$ and Raymond W. Ostelo ${ }^{\mathrm{c}, \mathrm{d}}$ \\ ${ }^{a}$ Medical Back Neck Centre, The Hague and Rugpoli Brabant Tilburg, the Netherlands \\ ${ }^{\mathrm{b}}$ Department of Rehabilitation, Medical Centre Alkmaar, Alkmaar, the Netherlands \\ ${ }^{\mathrm{c}}$ Department of Epidemiology and Biostatistics and the EMGO+Institute for Health and Care Research, VU \\ University Medical Centre, Amsterdam, the Netherlands \\ ${ }^{\mathrm{d}}$ Department of Health Sciences and the EMGO+ Institute for Health and Care Research, Faculty of Earth and \\ Life Sciences, VU University Amsterdam, the Netherlands \\ ${ }^{\mathrm{e}}$ Department of Neurosurgery, Medical Center Haaglanden, Gravenhage, the Netherlands \\ ${ }^{\mathrm{f}}$ The George Institute for Global Health, University of Sydney, Australia
}

\begin{abstract}
.
STUDY DESIGN: Prospective cohort study.

BACKGROUND: Although lumbar radiculopathy is regarded as a specific diagnosis, the most effective treatment strategy is unclear. Commonly used treatments include transforaminal epidural steroid injections (TESIs) and Mechanical Diagnosis \& Therapy (MDT), but no studies have investigated the effectiveness of this combination. MDT differentiates pain centralization (C) from non-centralization (NC), which indicates good vs. poor prognostic validity respectively.

OBJECTIVE: The main aims were 1) to determine changes in Mechanical Diagnosis and Therapy (MDT) pain response classifications after transforaminal epidural steroid injections (TESIs) in candidates for lumbar herniated disc surgery and 2) to evaluate differences in short and long term outcomes for patients with different pain response classifications.

METHODS: Candidates for lumbar herniated disc surgery were assessed with a MDT protocol and their pain response classified as centralizing or peripheralizing. For this study,only patients were eligible who showed a peripheralizing pain response at intake. All patients then received TESIs and were reassessed and classified using the MDT protocol, into groups according to pain response (resolved, centralizing, peripheralizing with less pain and peripheralising with severe pain). After receiving targeted treatment based on pain response after TESIs, ranging from advice, MDT or surgery, follow-up assessments were completed at discharge and at 12 months. The primary outcomes were disability (Roland-Morris Disability Questionnaire [RMDQ] for Sciatica), pain severity in leg (visual analogue scale [VAS], 0-100) and global perceived effect (GPE). Linear mixed-models were used to determine between-groups differences in outcome.

RESULTS: A total of 77 patients with lumbar disc herniation and peripheralizing symptoms were included. Patients received an average of 2 (SD 0.7) TESIs. After TESIs, 17 patients (22\%) were classified as peripheralizing with continuing severe pain.
\end{abstract}

\footnotetext{
* Corresponding author: Hans van Helvoirt, Van Schoonbekestraat 115, 2018 Antwerpen, Belgium. Tel.: +31 65101 9644; E-mail: hans.van.helvoirt@telenet.be.
} 
These patients underwent surgery and were not further evaluated. Eleven (14\%) patients were classified as resolved, 37 (48\%) as centralizing with significant less pain, and $12(16 \%)$ as peripheralizing with significant less pain. None of these patients underwent surgery. Resolved and centralizer subgroups had better outcomes in terms of VAS and RMDQ than the non-operated peripheralizers at discharge and at 12 months. The succes rates (GPE) for the resolved, centralizing, and peripheralizing with less pain patients were $100 \%, 100 \%$ and $33 \%$ respectively at short term, and $100 \%, 92 \%$ and $50 \%$ respectively at long term.

CONCLUSION: After TESIs, a peripheralizing pain pattern changed to resolved or centralizing in $62 \%$ of the patients. For the non-operated patients, those with a centralising pattern after TESIs reported better pain and disability outcomes than those with peripheralizing pattern at short and long term.

Keywords. Herniated disc, transforaminal epidural injection, MDT, McKenzie

\section{Introduction}

Lumbar disc herniations are the most common source of sciatica (i.e. pain radiating below the knee) estimates of the annual prevalence range from 3\% [1] to $14 \%$ [2]. Surgery can provide immediate relief of symptoms, however research has shown that functional outcomes of lower risk, non-operative treatments are equal to those following lumbar disc surgery, although recovery takes longer [3].

For patients with a herniated disc and persistent disabling radicular pain, therapeutic epidural injections are an increasingly popular treatment option, aimed at diminishing pain, improving function and preventing surgery [4,5]. Injection techniques vary and can be divided into transforaminal, interlaminar, and caudal approaches. Several studies have evaluated the effectiveness of these injections, however interpretation of the results is hampered by heterogeneity with respect to injection therapy technique and the absence of a standard placebo intervention. In a recent review, Pinto et al. [4] assessed the effectiveness of epidural corticosteroid injections for sciatica (i.e. pain radiating below the knee) and concluded that they are only minimally effective in pain and disability reduction, and only for short-term. They found no difference when comparing the three different epidural injection approaches. Although epidural injections are frequently performed in patients with disabling radicular pain, there are currently no clear therapeutic recommendations regarding inclusion criteria, timing, preferred techniques and the number of injections [6]. Although its comparative effectiveness with respect to other approaches remains unclear, the transforaminal approach has potential advantages over interlaminar and caudal injections. The most important of these is that transforaminal injections offer a more-targeted approach, delivering steroid directly to the presumable inflamed nerve root under fluoroscopic guidance. In our clinical practice, patients with persistent disabling radicular pain due to herniated disc lesions are initially classified with respect to their pain response (i.e. centralizing or peripheralizing) using Mechanical Diagnosis and Therapy (MDT) [7]. Centralizing pain means that the most distal pain abolishes in response to certain postures and end-range movements. Peripheralizing pain means that distal symptoms are produced and/or distal symptoms are made worse. After classification, patients with a centralizing pain response are treated with MDT, because for 'centralizers' there is some evidence that MDT is more effective than other physical therapy approaches [8-11]. Patients with a peripheralizing pain response are treated with TESIs. A possible explanation for a peripheralizing pain response is that radicular pain is aggravated during the MDT assessment process due to irritation of an inflamed nerve root [12-14]. Following this, it seems reasonable to treat peripheralizers firstly with TESIs in order to target the presumably inflamed nerve root, and secondly with physical therapy, if necessary. It may be that a peripheralizing pain response is an indicator for a more intensive procedure like TESIs, as there is preliminary evidence that patients with a peripheralizing response have a less favorable prognosis than patients with a centralization response [15].

In our clinical experience, the pain response frequently changes from a peripheralizing pattern into a centralizing pattern after TESIs, although this observation has never been systematically documented. As far as we know, there is only one small retrospective case series that has described such a change [16]. In this study three patients with acute cervical radiculopathy and peripheralizing pain underwent cervical transforaminal or intralaminar epidural injections at one or two levels. After this procedure, the three patients were classified as centralizers with MDT and subsequently treated according to MDT principles with direction specific exercises and posture correction. The combination of injections followed by MDT resulted in rapid and drastic improvements. 
The aims of the present study were 1) to determine changes in MDT pain response classification after transforaminal epidural steroid injections (TESIs) in candidates for lumbar herniated disc surgery and 2) to evaluate differences in short and long term outcomes for patients with different pain response classifications following TESIs.

\section{Methods}

Data were prospectively collected in two time periods, from August 2008 to October 2008 and from November 2009 to March 2010. Patients were recruited in the Medical Back Neck Centre, outpatient private practices in The Hague and in Rugpoli Veluwe in Velp, The Netherlands. The Regional Medical Ethics Committee North Holland determined that formal ethical approval was not required, because the study involved only usual care and outcome measurements were consistent with daily practice. This study is the second analysis of these data. The first study was an observational investigation of the effectiveness of TESIs plus physical therapy for this cohort. The results of the patients with chronic low back and leg pain $(\geqslant$ 3 months) are reported elsewhere [17]. In the present study, a small subsample of patients with a shorter duration of back pain was also included.

\subsection{Inclusion and exclusion criteria}

All patients were surgery candidates. Determination of suitability for surgery was performed by neurosurgeons and orthopedic surgeons according to CBO (Dutch Institute for Healthcare Improvement) guidelines for surgery in herniated disc lesions [18]. Prior to undergoing surgery, eligible patients were offered participation in the study, which involved an MDT assessment and a combination of TESIs and MDT as appropriate. Inclusion criteria were; lower extremity pain with or without low back pain, a lumbar disc herniation confirmed by MRI, two or more positive neurological signs (i.e. sensory deficits, muscle deficits, diminished jerk reflexes) peripheralizing pain during two MDT diagnostic sessions. Exclusion criteria were; pregnancy, contraindication to the use of corticosteroids (e.g. allergy for corticosteroids) or fluoroscopy, and specific causes of LBP that were not directly related to herniated discs (e.g., (suspicion of) malignancy, fractures, spinal stenosis, severe cases of spondylolisthesis), and inability to speak Dutch or English. The MDT as- sessments were done by two diplomat MDT therapists and one credentialed MDT therapist. Each patient was assessed twice with a MDT protocol within 48 hours. Only patients who showed peripheralizing symptoms at both assessments were included. Research has shown good reliability for observing centralizing and peripheralizing responses when done by physical therapists with appropriate educational level in MDT [19,20]. All participating patients signed informed consent.

\subsection{Intervention}

After the initial MDT classification procedure, all patients received TESIs of $20 \mathrm{mg}$ dexamethasone and $0.5 \mathrm{cc}$ lidocaïne $2 \%$, under fluoroscopic guidance with contrast medium (Omni Pac 240) as prescribed by International Spinal Intervention Society (ISIS) guidelines [21]. If necessary, within 10 to 14 days an appointment was made for an extra injection. Segmental level was determined by MRI findings combined with clinical picture. All medical doctors $(n=4)$ involved in administering the TESIs were trained in, and followed ISIS guidelines [21]. If pain relief was greater than $50 \%$ on the visual analogue scale (VAS) leg or if the patient refused another injection, no further injection was provided. Doctors could give up to a maximum of four TESIs to optimize pain relief. All decisions were made in a shared decision-making process between the patient and the physician. Subsequent injections were administered with 2 weeks intervals.

\subsection{MDT reassessment to establish pain response classification}

Ten to 14 days after the last TESI, patients were re-evaluated and re-classified by a MDT therapist and treated according to their pain response classifications. The four treatment subgroups consisted of 1) resolved symptoms (i.e. no pain with repeated movements or sustained positions); these patients were given advice using MDT principles, based on balance between flexion and extension activities and posture correction 2) centralizing; these patients were treated according to MDT principles with direction specific exercises and posture correction, 3) peripheralizing and significant less pain compared to the situation before TESIs; these patients were given advice/information using MDT principles and advice to stay active, with respect for worsening leg pain, and 4) peripheralizing with ongoing high levels of pain and/or disability; this subgroup was expected to have an unfavorable prognosis with conservative management and recieved herniated disc surgery . 


\subsection{Outcome measures}

The primary outcome measures were leg pain, disability and global perceived effect (GPE). Leg pain was measured using a 100-millimeter VAS ranging from 0 (no pain) to 100 (worst imaginable pain) over the past 24 hours. Disability was measured using the Roland-Morris Disability Questionnaire for sciatica (RMDQs) [22]. Total RMDQs score is the sum of positive answers to all the questions and ranges from 0 (no disability) to 23 (maximum disability). GPE was measured using a 7 point Likert scale, which ranged from 1 to 7 . An a priori decision was made to dichotomize GPE into success (scores 1 and 2; complete and nearly complete recovery) and no success (scores 3 to 7 ; slight recovery to worse than ever).

Secondary outcome measures were back pain, anxiety and depression. Back pain was measured with a 100-millimeter VAS ranging from 0 (no pain) to 100 (worst imaginable pain) over the last 24 hours. Anxiety and depression were measured with the Hospital Anxiety and Depression Scale (HADS) [23]. The HADS contains two subscales of 7 items for depression and 7 items for anxiety. Each item is rated on a four-point scale, giving maximum scores of 21 for anxiety and depression.

Other data gathered were age, sex, duration of the complaints, number of TESIs, number of MDT treatment sessions, complications due to any of the study treatments, other treatments during the follow-up period including lumbar surgery, medication use and work status. Data were collected at intake, at discharge after MDT and 12 months after intake.

\subsection{Statistical analysis}

Baseline characteristics of the patients (i.e. before TESIs) were calculated for each of the four classifications. Frequencies and percentages of patients in each pain response classification subgroup after TESIs were calculated. Fisher exact, Mann-Whitney and ttests were used to test differences in baseline characteristics between patients who eventually underwent surgery with those that did not receive surgery.

To explore differences in short and long term outcomes between the three non-surgery subgroups (resolved, centralizing and peripheralizing and significantly less pain) linear mixed model analyses were used for continuous primary and secondary outcomes, controlling for baseline scores and sex. For each subgroup, regression coefficients with $95 \%$ confidence in- tervals (CI) between baseline and follow-up measurements were calculated. The success rates for each subgroup at short and long term (GPE) were compared with a chi-square test (likelihood ratio (LR)).

For patients who underwent surgery only short term effects were available. For this subgroup paired t-tests were used to calculate differences before and after TESIs.

For all tests $p<0.05$ was considered significant. The data were analysed using IBM SPSS Statistics Version 20.0 (IBM Corporation, Armonk, NY, USA)

\section{Results}

A total of 132 potentially eligible participants were identified of which 79 patients met all inclusion criteria. The reasons for ineligibility were: centralizing pain response at initial MDT assessments ( $n=29$ on the first visit, and $n=12$ on the second visit), insufficient understanding of English or Dutch language $(n=4)$, refused injections $(n=5)$ and undefined reasons $(n=$ $3)$. Of the 79 patients who participated and were injected, 2 patients dropped out: one moved to another address and did not want to continue participation and one refused further cooperation, no follow-up data was available for these patients. This resulted in a study sample of 77 patients (see Fig. 1).

The mean age for all 77 patients was 47.1 years (standard deviation [SD] 9.2), the mean VAS leg pain was $58.1 \mathrm{~mm}$ (SD 25.1) and the mean RMDQs score was 14.2 (SD 5.0). In the sample 51.9\% were men and $91 \%$ had chronic low back pain ( $\geqslant 3$ months). Patients were injected with an average of 2 (SD 0.7) TESIs. The number of patients in each of the four classifications after TESIs was; resolved $14 \%(n=11)$, centralizing $48 \%$ ( $n=37)$, peripheralizing with significant less pain $16 \%(n=12)$ and peripheralizing with ongoing high levels of pain and/or disability $22 \%(n=17)$. The patients in the last subgroup underwent surgery. Table 1 presents baseline characteristics (before TESIs) amongst the patients eventually categorised in the different pain response subgroups. All patients with centralizing symptoms reported significantly less pain, except one. This patient was later categorized in the subgroup peripheralizing with ongoing high levels of pain and/or disability and underwent surgery after half a year. 
Table 1

Baseline characteristics before injections amongst the different pain response groups $(n=77)$

\begin{tabular}{|c|c|c|c|c|}
\hline Characteristics & Resolved & Centralizing & $\begin{array}{c}\text { Peripheralizing with } \\
\text { less pain }\end{array}$ & $\begin{array}{c}\text { Peripheralizing with } \\
\text { continuing pain }\end{array}$ \\
\hline Number $(\%)$ & $11(14 \%)$ & $37(48 \%)$ & $12(16 \%)$ & $17(22 \%)$ \\
\hline Age in years & $45.5(13.7)$ & $46.7(8.3)$ & $49.8(10.5)$ & $46.9(6.8)$ \\
\hline Male, $\%$ & 54.5 & 64.9 & 16.7 & 47.1 \\
\hline Lower back surgery, $\%$ & 0 & 2.7 & 0 & 5.9 \\
\hline Duration of current LBP in months $\dagger$ & $6.0(4.0-17.0)$ & $9.0(3.5-16.0)$ & $7.5(4.5-45.0)$ & $5.5(4.0-21.0)$ \\
\hline Current LBP (0-5 weeks), \% & 0 & 5.4 & 0 & 0 \\
\hline Current LBP (6-12 weeks), \% & 0 & 8.1 & 8.3 & 6.3 \\
\hline Current LBP $(>12$ weeks $), \%$ & 100.0 & 86.5 & 91.7 & 93.7 \\
\hline Continuous pain in the lower back, $\%$ & 45.5 & 58.8 & 58.3 & 58.8 \\
\hline Continuous pain in the leg, $\%$ & 36.4 & 58.8 & 75.0 & 64.7 \\
\hline Currently taking medication for LBP, $\%$ & 63.6 & 56.8 & 41.7 & 82.4 \\
\hline Pain in sitting, $\%$ & 63.6 & 73.5 & 81.8 & 88.2 \\
\hline Employed, \% & 100 & 97.1 & 91.7 & 75 \\
\hline Employed and currently working, $\%$ & 54.5 & 62.9 & 41.7 & 6.3 \\
\hline $\begin{array}{l}\text { Employed, but currently working less hours } \\
\text { or lighter duty, } \%\end{array}$ & 0 & 2.9 & 8.3 & 6.3 \\
\hline RMDQs $(0-23)$ & $11(5.9)$ & $14.2(4.6)$ & $12.5(4.4)$ & $17.5(3.7)$ \\
\hline Pain leg $(0-100)$ & $49.9(28.2)$ & $55.6(22.1)$ & $48.8(31.5)$ & $75.6(16.6)$ \\
\hline Pain lower back $(0-100)$ & $40.0(25.4)$ & $45.5(24.8)$ & $37.3(19.8)$ & $60.8(29.9)$ \\
\hline HADSA $(0-21)$ & $2.4(1.1)$ & $5.2(3.1)$ & $5.2(3.9)$ & $7.6(4.1)$ \\
\hline HADSD $(0-21)$ & $2.7(1.8)$ & $5.2(3.0)$ & $5.6(3.5)$ & $7.7(3.9)$ \\
\hline
\end{tabular}

Values are the mean (standard deviation) unless otherwise indicated.†Median and inter-quartile range. Abbreviations: LBP, low back pain, RMDQs, Roland-Morris Disability Questionnaire for sciatica; HADSA, Hospital Anxiety Depression Scale for Anxiety; HADSD, Hospital Anxiety Depression Scale for Depression.

\subsection{Comparison between patients that received surgery and those that did not}

At baseline, the 17 patients who underwent surgery after TESIs scored statistically significantly higher on pain in the leg $(p<0.001)$, disability $(p<0.001)$, pain in the back $(p=0.01)$, anxiety $(p=0.003)$ and depression $(p=0.002)$ compared to the non-surgery patients. Patients who underwent surgery were also less likely to be employed ( $p=0.018$ ).

\subsection{Responders to TESIs}

In this study, 60 patients $(78 \%)$ responded well to TESIs and were classified after receiving TESI in one of the three subgroups: resolved, centralizing and peripheralizing with significantly less pain. During the one-year follow-up time, none of these patients underwent surgery and only a minority of the patients received additional conservative treatment elsewhere $(19.3 \%)$, mostly graded activity training. Use of pain medication diminished from $55 \%$ at baseline, to $10 \%$ at discharge (between 4 to 7 weeks) and 6\% at one year (long term). For employed patients, $41 \%$ had work restrictions at baseline, this reduced to $16 \%$ at discharge and $10 \%$ at long term.

\subsection{Differences between the three types of responders to TESIs}

On average, the number of MDT sessions following TESIs in the resolved subgroup was 1.2 (SD 0.4), 3.6 (SD 1.4) in the centralizer subgroup, and 2.7 (SD 1.7) in the peripheralizing with significant less pain subgroup. Patients in the resolved and centralizing pain subgroups reported statistically significant improvements compared with baseline on the primary outcomes leg pain and disability at short and long term (Table 2, all $p<0.001$ ). Patients classified as peripheralizing with significant less pain reported statistically significant improvements for pain in the leg and disability at long term $(p<0.001)$, but not at short term.

The effects of treatment were larger in the centralizer subgroup compared to the peripheralizing with less pain subgroup at short term (mean difference VAS $29.4,95 \%$ CI 13.6 to $45.2, p<0.001$ and mean difference RMDQs $6.7,95 \%$ CI 3.6 to $9.8, p<0.001)$ ) and long term (mean differences VAS 21.2, 95\% CI 2.1 to 40.3, $(p<0.05)$ and RMDQs $4.8,95 \%$ CI 1.2 to 8.4 $(p<0.05))$ (Table 2). For secondary outcomes, results in the resolved and centralizer subgroups were also significantly better compared to the peripheralizing with less pain subgroup on the short and long term (Table 2). For primary and secondary outcomes, there were no 


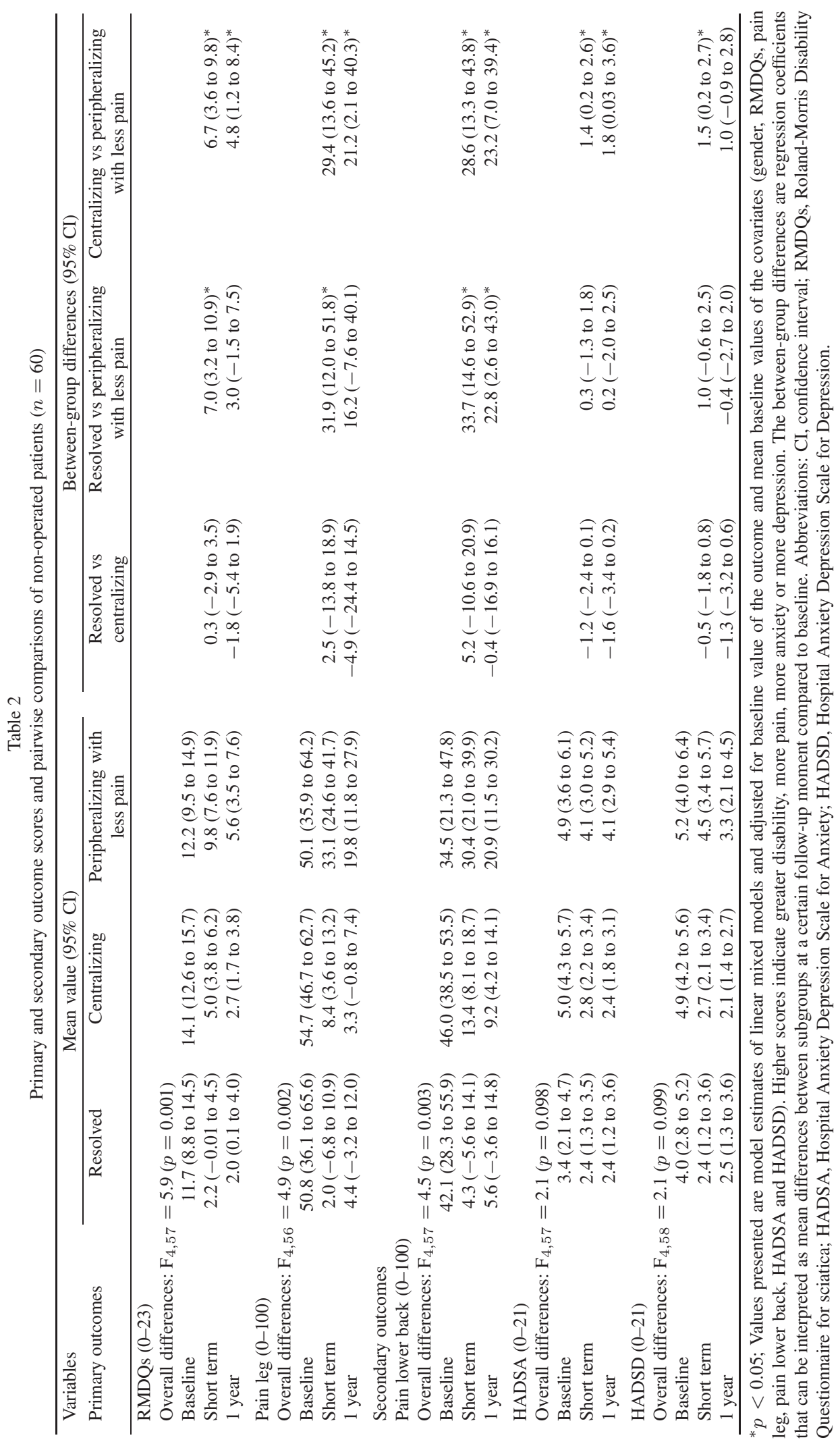


statistically significant differences in change between the resolved and centralizer subgroups.

The succes rates (GPE) for the subgroups resolved, centralizing and peripheralizing with less pain were $100 \%, 100 \%$ and $33 \%$ respectively, and at long term $100 \%, 92 \%$ and $50 \%$ respectively (Table 3 ). The differences between success and non-success were statistically significant different between the three subgroups at short term $\left(\mathrm{LR} \mathrm{X} \mathrm{X}^{2}=31.8\right.$ exact $\left.p<0.001\right)$ and long term $\left(\mathrm{LR} \mathrm{X} \mathrm{X}^{2}=11.7\right.$, exact $\left.p=0.002\right)$.

\subsection{Non responders on TESIs}

All 17 patients classified as peripheralizing with ongoing high levels of pain and/or disability underwent lumbar discectomy; 16 patients in the weeks after TESIs and 1 patient after half a year. Although these patients did not experience a clinically relevant effect from TESIs, there was a statistically significant improvement in pain in the leg, pain in the back, anxiety and depression after TESIs (Table 4). No long-term follow-up measurements were collected for these patients.

\section{Discussion}

This is the first prospective study that assessed changes in MDT pain response classification after TESIs in patients who were candidates for lumbar herniated disc surgery. Changes in pain response classification (from peripheralizing to centralizing or resolved) were identified in $62 \%$ of the patients. Following TESIs, a subgroup was classified as resolved (14\%) and a large minority (48\%) as centralizers with significant less pain. We think this change in pain pattern after TESIs is due to reduction of inflammation around the nerve root. We hypothesize that this inflammation was obstructing reduction of the mechanical displacement by MDT before the TESIs. We could not extract any pre-TESI clinical, mechanical, or imaging predictors of such a conversion from this data.

In addition to a change in classification, these patients experienced good symptomatic outcome at short and long term. The good outcomes in the centralizing subgroup are in accordance with studies that report good outcomes for centralizers treated with MDT [9, 24,25]. The outcomes of the present study justify some optimism, especially when recognizing that all included patients had peripheralizing pain responses, MRI-verified herniated discs that corresponded to clin- ical assessment and $91 \%$ of the patients had chronic symptoms.

A third subgroup (16\%) was classified as peripheralizing with significant less pain. These patients did not undergo surgery during the one year follow-up, but had less favourable outcomes compared to the resolved and centralizer subgroups. Our findings in relation to this subgroup differ from those of Albert et al. [12] They studied patients with severe sciatica and radiculopathy and found that patients with centralizing and periferalizing pain responses improved equally. Interestingly, they classified $7.9 \%$ of patients in a no-effect subgroup. Their no-effect subgroup was decribed as having no change in pain and or location during initial MDT assessment. This subgroup had poorer outcome. None of the patients assessed for our study were classified as no-effect, all had some symptomatic response to the MDT assessment. Possibly this is due to differences in repeated movement testing. In contrast to Albert et al. we did not restrict movement in a certain direction to 10 repetitions. The assessment continued up to 40 repetitions with the addition of therapist overpressure if needed. Albert et al. also used only one single assessment to classify the patients whereas we classified the patients twice within 48 hours. A study of Werneke et al. [24] showed that centralization is sometimes not identified in one visit, this aligns with our findings where 12 of the 41 patients showed a centralization reponse in the second visit, but not in the first.

A fourth subgroup (22\%) was classified as peripheralizing with ongoing high levels of pain and/or disability. The unfavorable outcome following TESIs and the ongoing pain and disability meant that these patients were not given further conservative treatment but referred for surgery. Although outcome measurements after TESIs showed statistically significant change in leg pain and disability, the differences were not deemed clinically relevant by the patients. These findings are in line with Skytte et al. [25] who found that patients with peripheralizing response were six times more likely to undergo surgery than centralizers. Interestingly, the patients who were operated showed higher levels of pain and disablity at intake compared to the non-surgery patients. It is noted that our study recruited only patients with peripheralizing symptoms, our results may not be directly comparable with other studies [26].

Our results differ from the results of two large prospective studies. These studies also used MDT classifications and treatment according to MDT principles and found no prognostic value of pain patterns clas- 
Table 3

General perceived effect at short and long term of non-operated patients $(n=60)$

\begin{tabular}{lccc}
\hline General perceived effect (success, \%) & Resolved & Centralizing & Peripheralizing with less pain \\
\hline Short term & 100 & 100 & 33 \\
1 year & 100 & 92 & 50 \\
\hline
\end{tabular}

Table 4

Primary and secondary short term outcomes of patients who underwent surgery $(n=17)$

\begin{tabular}{lccc}
\hline Variables & Baseline & Short term & $\mathrm{p}^{*}$ \\
\hline RMDQs (0-23) & $17.5(3.7)$ & $15.8(5.1)$ & 0.11 \\
Pain leg (0-100) & $75.6(16.6)$ & $61.7(24.6)$ & 0.03 \\
Pain lower back (0-100) & $60.8(29.9)$ & $48.7(28.5)$ & 0.05 \\
HADSA (0-21) & $7.6(4.1)$ & $6.8(4.1)$ & 0.03 \\
HADSD (0-21) & $7.7(3.9)$ & $6.9(3.9)$ & 0.04 \\
\hline
\end{tabular}

The $\mathrm{p}$ value refers to differences between baseline (before injections) and short term (after injections). Abbreviations: RMDQs, Roland-Morris Disability Questionnaire for sciatica; HADSA, Hospital Anxiety Depression Scale for Anxiety; HADSD, Hospital Anxiety Depression Scale for Depression.

sification. Schmidt et al. [27] prospectively followed 793 patients with low back pain (duration 4-26 weeks) of whom $63 \%$ had leg pain. They classified patients as centralizers (18\%), non lasting centralizers $(21 \%)$, peripheralizers $(13 \%)$, and no change (48\%). All subgroups improved significantly to a clinically important degree over one year. Christiansen et al. [28] studied 331 low back pain patients who were sick listed for 4-12 weeks. They classified patients as centralizers $(30 \%)$, peripheralizers $(8 \%)$ and no response $(62 \%)$. Of the 122 patients with radiculopathy, $20.5 \%$ were operated in the following year, which is similar to our study, but they were evenly distributed along the different subgroups. However, results of these studies cannot directly be compared with our study, because we only included patients with peripheralizing symptoms and treated patients with TESIs in combination with MDT. The studies of Schmidt et al. and Christiansen et al. did not use TESIs and only used MDT or other physical therapy strategies.

Due to this conflicting evidence there is still uncertainty if centralization and peripheralization are prognostic factors regardless of treatment, or effect modifiers related to a specific treatment.

As we studied the combined effect of treatment with TESIs and MDT, we can't draw conclusions about the effect of the single interventions, but we have shown that TESIs could change pain patterns as recognized with MDT. In that sense TESIs made it possible to use direction specific exercises, which were not possible before TESIs. Our study showed in general good long term outcomes which was not shown in the review of Pinto et al. [4] who assessed the effectiveness of epidural corticosteroid injections as a standalone intervention.

\subsection{Limitations}

For the patients who did undergo surgery, only baseline and outcomes after TESI's were available. Therefore, it was not possible to make comparisons between the outcomes of this subgroup with the other subgroups. This study lacks an untreated control group, so it is not clear what proportion of patients would have changed classification simply with the passage of time. The study was not randomised, so definitive conclusions about effects cannot be drawn. Confirmation of the study is necessary due to a rather small sample size.

\section{Conclusion}

This study provides preliminary evidence that TESIs can convert patients with periferalizing pain patterns to centralizing pain patterns or no pain. The results indicate that this combined treatment prevents surgery and shows long term effect in a substantial proportion of candidates for herniated disc surgery. We believe that a change to a centralizing pain pattern can be influential in directing follow-up conservative treatment, as patients with centralizing pain patterns can be treated with direction-specific exercises, which is not possible in patients with periferalizing pain patterns. For the non-operated patients the prognosis of peripheralizers after TESIs was less favorable compared to centralizers and resolved subgroups.

\section{Acknowledgements}

D. de Haan, MD, F. van de Vet, MD, M. Schepers, MD, M. Eckhaus, MD, H. Meihuizen, PT, L. Geerlings, PT. 


\section{References}

[1] Younes M, Bejia I, Aguir Z et al. Prevalence and risk factors of disk-related sciatica in an urban population in Tunisia. Joint Bone Spine 2006 October;73(5): 538-542.

[2] Palmer KT, Griffin MJ, Syddall HE, Pannett B, Cooper C, Coggon D. The relative importance of whole body vibration and occupational lifting as risk factors for low-back pain. Occup Environ Med 2003 October;60(10): 715-721.

[3] Peul WC, Van Houwelingen HC, van den Hout WB et al. Surgery versus prolonged conservative treatment for sciatica. N Engl J Med 2007 May 31;356(22): 2245-2256.

[4] Pinto RZ, Maher CG, Ferreira ML et al. Epidural corticosteroid injections in the management of sciatica: a systematic review and meta-analysis. Ann Intern Med 2012 December 18;157(12): 865-877.

[5] Arts MP, Peul WC. Timing and minimal access surgery for sciatica: a summary of two randomized trials. Acta Neurochir (Wien ) 2011 May;153(5): 967-974.

[6] Koes BW, Van Tulder MW, Peul WC. Diagnosis and treatment of sciatica. BMJ 2007 June 23;334(7607): 1313-1317.

[7] McKenzie R, May S. The lumbar spine. Mechanical diagnosis \& therapy (volume 1\&2). 2nd ed. Waikanae: Spinal Publications New Zealand Ltd; 2003.

[8] Long A, Donelson R, Fung T. Does it matter which exercise? A randomized control trial of exercise for low back pain. Spine (Phila Pa 1976 ) 2004 December 1;29(23): 2593-2602.

[9] Werneke MW, Hart DL, Cutrone G et al. Association between directional preference and centralization in patients with low back pain. J Orthop Sports Phys Ther 2011 January;41(1): 22-31.

[10] Browder DA, Childs JD, Cleland JA, Fritz JM. Effectiveness of an extension-oriented treatment approach in a subgroup of subjects with low back pain: A randomized clinical trial. Phys Ther 2007 December;87(12): 1608-1618.

[11] Petersen T, Larsen K, Nordsteen J, Olsen S, Fournier G, Jacobsen S. The McKenzie method compared with manipulation when used adjunctive to information and advice in low back pain patients presenting with centralization or peripheralization. A randomized controlled trial. Spine (Phila Pa 1976) 2011 February 24.

[12] Albert HB, Manniche C. The efficacy of systematic active conservative treatment for patients with severe sciatica: A single-blind, randomized, clinical, controlled trial. Spine (Phila Pa 1976) 2012 April 1;37(7): 531-542.

[13] Donelson R. Mechanical diagnosis and therapy for radiculopathy. Phys Med Rehabil Clin N Am 2011 February;22(1): 75-89.

[14] Desai MJ, Shah B, Sayal PK. Epidural contrast flow patterns of transforaminal epidural steroid injections stratified by commonly used final needle-tip position. Pain Med 2011 June;12(6): 864-870.

[15] May S, Aina A. Centralization and directional preference: A systematic review. Man Ther 2012 June 11.

[16] Desai MJ, Padmanabhan G, Simbasivan A, Kamanga-Sollo GG, Dharmappa A. Directional preference following epidural steroid injection in three patients with acute cervical radiculopathy. Pain Pract 2013 September;13(7): 559-565.

[17] van Helvoirt H, Apeldoorn AT, Ostelo RW et al. Transforaminal epidural steroid injections followed by Mechanical Diagnosis and Therapy to prevent surgery for lumbar disc herniation. Pain Med 2014;15: 1100-1108.

[18] Dutch institute for Health Care Improvement (CBO). Richtlijn lumbosacraal radiculair syndroom [Guideline lumbosacral radicular syndrome]. Utrecht: CBO; 2008.

[19] Kilpikoski S, Airaksinen O, Kankaanpaa M, Leminen P, Videman T, Alen M. Interexaminer reliability of low back pain assessment using the McKenzie method. Spine (Phila Pa 1976 ) 2002 April 15;27(8): E207-E214.

[20] Werneke M, Hart DL, Cook D. A descriptive study of the centralization phenomenon. A prospective analysis. Spine (Phila Pa 1976 ) 1999 April 1;24(7): 676-683.

[21] Bogduk Ne. Practice Guidelines for Spinal Diagnostic and Treatment Procedures. 2nd ed. San Francisco: International Spinal Intervention Society; 2013.

[22] Patrick DL, Deyo RA, Atlas SJ, Singer DE, Chapin A, Keller RB. Assessing health-related quality of life in patients with sciatica. Spine (Phila Pa 1976 ) 1995 September 1;20(17): 1899-1908.

[23] Zigmond AS, Snaith RP. The hospital anxiety and depression scale. Acta Psychiatr Scand 1983 June;67(6): 361-370.

[24] Werneke M, Hart DL. Centralization phenomenon as a prognostic factor for chronic low back pain and disability. Spine (Phila Pa 1976 ) 2001 April 1;26(7): 758-764.

[25] Skytte L, May S, Petersen P. Centralization: its prognostic value in patients with referred symptoms and sciatica. Spine (Phila Pa 1976 ) 2005 June 1;30(11): E293-E299.

[26] Riew KD, Yin Y, Gilula L et al. The effect of nerve-root injections on the need for operative treatment of lumbar radicular pain. A prospective, randomized, controlled, double-blind study. J Bone Joint Surg Am 2000 November;82-A(11): 15891593.

[27] Schmidt I, Rechter L, Hansen VK, Andreasen J, Overvad K. Prognosis of subacute low back pain patients according to pain response. Eur Spine J 2008 January;17(1): 57-63.

[28] Christiansen D, Larsen K, Jensen OK, Nielsen CV. Pain response classification does not predict long-term outcome in patients with low back pain who are sick-listed. J Orthop Sports Phys Ther 2010 October;40(10): 606-615. 